\title{
A review of human skin detection applications based on image processing
}

\author{
Hussein Ali Hussein Al Naffakh ${ }^{1}$, Rozaida Ghazali ${ }^{2}$, Nidhal Khdhair El Abbadi ${ }^{3}$, Ali Nadhim Razzaq ${ }^{4}$ \\ ${ }^{1,2,4}$ Faculty of Computer Science and Information Technology, Universiti Tun Hussein Onn Malaysia, Malaysia \\ ${ }^{3}$ Faculty of Education, University of Kufa, Iraq
}

\section{Article Info}

Article history:

Received Apr 29, 2020

Revised Jun 1, 2020

Accepted Jul 18, 2020

\section{Keywords:}

Applications of human skin

Dataset

Skin color modeling

Skin detection

ABSTRACT

In computer science, virtual image processing is the use of a digital computer to manipulate digital images through an algorithm for many applications. To begin with a new research topic, the must trend application that gets many requests to develop should know. Therefore, many applications based on human skin and human life are reviewed in this article, such as detection, classification, blocking, cryptography, identification, localization, steganography, segmentation, tracking, and recognition. In this article, the published articles with the topic of human skin-based image processing are investigated. The international publishers, such as Springer, IEEE, arXiv, and Elsevier are selected. The searching is implemented with the duration criteria of 2015-2019. It noted that human skin detection and recognition are the most repetitive articles with $43 \%$ and $28.5 \%$, respectively of the total number of the investigated articles. The usage of human skin models is being widely used in the image processing of various applications.
\end{abstract}

This is an open access article under the CC BY-SA license.

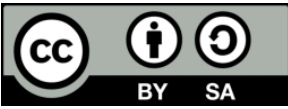

\section{Corresponding Author:}

Hussein Ali Hussein Al Naffakh,

Faculty of Computer Science and Information Technology,

Universiti Tun Hussein Onn Malaysia,

86400 Parit Raja, Johor, Malaysia.

Email: aulanone@gmail.com

\section{INTRODUCTION}

Skin detection plays an important role in a wide range of image processing applications ranging from recognition, detection, tracking, classification, segmentation, identification, blocking, localization, cryptography, steganography, and content-based image retrieval (CBIR) systems. Nowadays, the image pressing for human skin detection based on skin color recognition has demonstrated an attractive, efficient, powerful technique and partial occlusions. For this application, the skin color recognition can be work simultaneously with other methods such as; shape, boundary, geometry, and other features are used to improve the accuracy of the system. An accurate system of detection; it has seven regions (feature points, skin tone, face, diseases, pornographic, hand, and adult). For recognition, it has pornography, disease, face, gender and hand. Tracking application has face, diseases, and hand. Segmentation application has face, diseases and hand. Steganography has skin tone and diseases. Localization application has hand region only. Identification also has for adult only. Cryptography application has diseases. Blocking application has adult. Classification has face, hand, diseases and age [1-9].

The skin detection based on color information is the technique to identify the skin and non-skin regain of the tested image. The skin regains means any part of the human skin that noted in the image under the test of the human skin detection system, such as the face, legs, and hands. Therefore, the skin detection is the first process of any recognition system such as face recognition [10-15]. This study aims to investigate 
the up to date researches of human skin detection applications. This article tried to sum up the differences between these applications, their strengths, and limitations.

This article is organized as follows: section 2 illustrates the human skin detection process such as recognition, detection, tracking, classification, segmentation, identification, blocking, localization, and cryptography. Section 3 demonstrates the desiccation and future work of this application. Finally, section 4 concludes the presented techniques and recommended the futures of human skin detection using image processing.

\section{HUMAN SKIN APPLICATION}

The detection of the skin plays an important role in a wide range of image processing applications, from facial recognition to face tracking and various digital communication domains. There are ten types of human skin applications, which are detection, classification, blocking, cryptography, identification, localization, steganography, segmentation, tracking and recognition, as shown in Figure 1. For the next section, we explain each application and its correlated datasets.

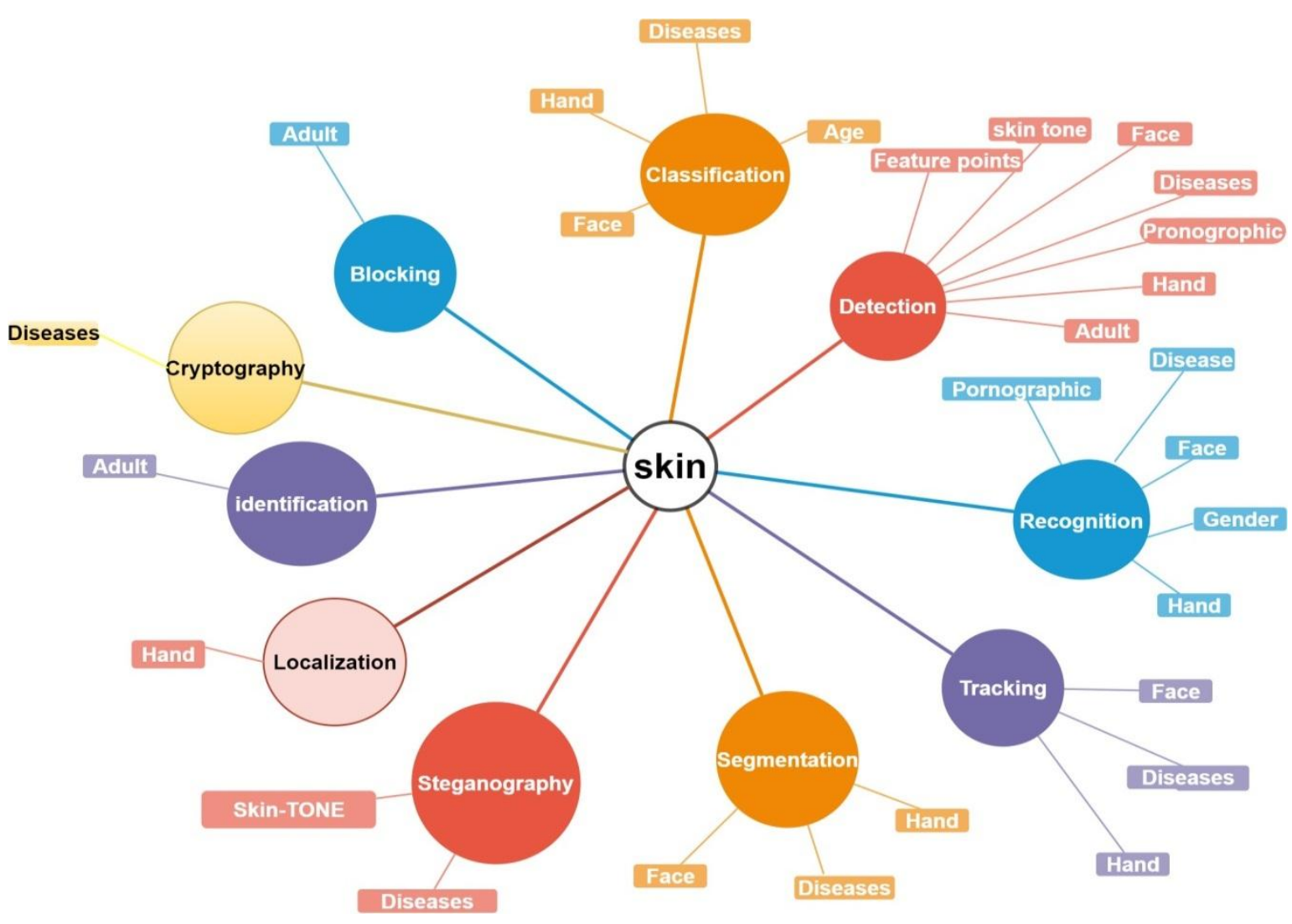

Figure 1. Types of human skin applications

\subsection{Recognition}

Hand gesture recognition helps us to contact people with disabilities by using sign language alphabet and controlling some robots and appliances without a controller. In human computer interface ( $\mathrm{HCI})$, gesture recognition is an important topic which needs to have a breakthrough. It has been used in combination with skin detection in more than 17 research like [1-3]. DATA: ECHO, personal data, NITS hand gesture, VGG16, collected from a project supported by the polish ministry of science \& higher education. The test data are collected from videos captured by web-camera, collected a dataset of twenty-four. New dataset is created in which the signs from www.indiansignlanguage.org are replicated, Indian sign language (ISL) gestures.

Face recognition is an important problem when the photo of a suspect is not available or is captured with very poor quality. It has been used in combination with skin detection in more than 14 research studies like deep convolutional neural networks (DCNN) [4], deep neural network [5], recognition of skin from hyperspectral face [6]. DATA: (facial spectral, CMU, VidTIMIT, AT\&T \& ORL, ORL, LCPTS Facial \& 
Caltech, LFW, from internet, FEI, LFW, Caltech \& FERET, IIITD Viewed, CUFS, e-PRIP \& MGDB Unviewed, pictures captured by laptop web camera, FERET, CASIA-WebFace\&FaceScrub).

Subsequent work has commenced to analyze the feasibility of the state-of-the-art to the recognition of image within the domains of dermascopy and dermatology. There are seven research studies available on skin diseases recognition for cancer, Psoriasis Skin, Skin Lesion, and Skin Lesion as [7, 8, 16, 17].

An overview of the dataset containing all points to present an identification challenge for melanoma was selected at the international symposium on biomedical imaging (ISBI 2016). In order to carry out the trace and evaluation, we used the data set published by the international collaboration on skin imaging (ISIC) to challenge the international symposium on biomedical imaging 2016 (ISBI 2016) entitled "Skin Lesion Analysis toward Melanoma Detection".

In recent years it has been suggested that people's demographic characteristics, such as age and gender orientation have been considered in the past decade [18]. Automated images face recognition is implemented by using three supervised learning machines, which are adobos, neural network, and SVM. Recognizing demography characteristics of individuals, for example, age and gender using automatic image recognition taken much consideration in last few years [18]. DATA: (FERET). In order to identify obscene images on the Web, the two key points are the accuracy and speed of identification. At present, many techniques are recommended to recognize the obscene images. One of the functions of skin detection is the identification of obscene images and pornography such as; multimedia files pre-processed, to analyze skin-colored pixels [19], Oriented FAST and Rotated BRIEF (ORB) [20], and combination of global and local features [21]. DATA: (From internet, NPDI).

\subsection{Detection}

Skin cancer is a major medical problem in general. If it can be detected timely then it can save life costs. Melanoma skin cancer is one of the most fatal skin cancer forms but can be easily cured if detected early enough. More than 23 researches had presented a solution for detecting early-stage melanoma skin cancer and detection of skin lesions like automated classification using deep neural networks [22], algorithms for automated diagnosis of melanoma [23], selection of a classifier [24], deep convolutional neural networks [25]. DATA: (PH2, ISIC, ISBI, Personal data, Edinburgh, Synthesis, from internet, DermIs, DermQuest).

Face detection is used in many fields, such as: intelligent video surveillance, face recognition, mobile photography equipment, etc. These applications require skin detection to achieve face detection quickly. These have been used in combination with skin detection in around 33 studies like color matching [26], three dimensional model of the human face using optimization-based reconstruction algorithms [27], swarm of particles in a kernel [28], k-nearest neighbors (KNN) algorithm and high boost filter [29]. DATA: (Personal data, FEI, Pointing'04, Bao Face, Group, Utrecht, MIT, AFW, Wider Face, ATM, PIE, FERET, LFW, Caltech, CMU, HHI, VidTIMIT, AT\&T, ORL, M2VTS, XM2VTS, VALID, Helen, Faces in the, Wild, Microsoft hand gesture, Padua Hand gesture, Padua Face Dec., FEI, images of the same person, from the video, from internet, from the video dataset ChokePoint, Paratheepan, FDDB, Pascal Faces, Custom, pictures captured by laptop web camera, MIT-CBCL, Georgia Tech, University of Oulu Physics-based face, CMUPIE, IMM).

A powerful detection of the hand signals and gestures remained challenging test for the basic computer, which was under real conditions. It may be useful to identify some of the applications in the images, such as communication that was recorded through a sign language analysis. Hand detection is mandatory for some hand-related functions, such as understanding hand gesture and pose, which is very useful for mechanical technology like robotics and interaction between humans and computers. The hand detection of CNN [30], CNN [31], multiple scale region-based fully convolutional networks [32], CNN [33], multi-feature CNN [34] are based on detecting skin pixels. DATA: (Oxford, Egohands, Personal dataset, 5-signer, VIV, Egohands, HGR).

The scale of adult content on the Internet is increasing every day. Many of the obscene and pornographic stuff is unlimited and open to all customers and users. The Adult based Page detection plays an important role in preventing sexual entertainment and pornography. In the area of adult image detection, noteworthy articles have been written on the detection of porn images with skin color CNN and RNN [28] forward an approach [29]. DATA: (From internet, NPDI).

With the growing amount of inappropriate content on the Internet like pornography the need to detect and filter such material has arisen. They [35], color-saliency preserved mixture deformable part model [36], deep convolutional neural networks [37], key-frame extraction algorithm [38], local feature with Bagof-Words models (BoW) [39], deep learning [40], deep learning [41], optimized nudity recognition [19] are proposed pornographic detection methods based on low level features of skin regions. DATA: (Video data, Pornography-800 \& Pornography-2k, APPA-Real, from Internet, NPDI) 


\subsection{Tracking}

An important area of research in computer vision is target tracking. It is usually applied in the area of in artificial intelligence (AI), for example, reorganization, surveillance, intelligent traffic, automatic control, and human-computer communication, etc. [42]. The tracking of face is an important research in the area of analysis of image and computer Vision framework. As a result of face tracking help security forces to investigate the wrongdoing and crimes. Analysts developed a technique for facial tracking based on autonomy-oriented entities (AOEs), and deep learning concepts [43], SDN algorithm [44-47] to highlight skin color, and extraction. DATA: (APIDIS, from video, Personal data, Visual Tracker Benchmark, images of the same person).

Hand gesture recognition system can be used for human-computer interaction (HCI). Depending on the skin's detection, some researchers used Hand gesture recognition [48-50]. DATA: (Grabbing hand gesture, Face zoom out, Occluded hands, Personal data, ECHO).

\subsection{Classification}

Skin disease is one of the most common illnesses in human daily life when people want their first assessment, there is no doubt that the VRS visual recognition system is helpful even if not perfect. For example, if a detailed section of skin disease has been performed, the user can bring up a recent picture of skin disease for diagnosis. Surprisingly, most research has been conducted using artificial vision technology to identify common skin diseases using common sense. There are many applications for skin disease, skin cancer, Skin Lesions, and skin pigmentation disorders [51-54]. DATA: (Edinburgh \&MED-NODE, Personal data, ISBI, PH2, ISIC, the ISIC Archives, the Edinburgh Dermofit, Image Library).

Hand gestures and motions are commonly used in major applications such as human machine interface design, sign languages, virtual reality, and animation, etc. The accuracy of a consistent gesture sequence is based on the technology used to extract the tasks used in the system. From the classification of the skin, it was observed that the area was used to determine the hand boundary [32, 55]. DATA: (Oxford, Sebastien Marcel, Cambridge Hand Gesture, NUS Hand Posture).

Facial skin assessment is crucial for a number of fields including the make-up industry, dermatology and plastic surgery [56]. DATA: DermNet. With the rapid development of the internet, obscene and pornographic images/videos can more easily spread and cause greater harm to the social stability and teenagers' mental health [57]. DATA: from Internet. Human age classification via face images is not only difficult for human beings but also challenging for a machine. Facial skin ageing features consist of facial skin textural features and wrinkle analysis [58]. DATA: FG-NET ageing.

\subsection{Segmentation}

The segmentation components of the system are used to identify the lesion areas and Melanoma (cancer) for skin on the medical images [53,59-63]. DATA: FG-NET ageing. Hand segmentation for handobject interaction is a necessary pre-processing step in many applications such as augmented reality, medical application, and human-robot interaction [64-67]. DATA: (ECHO, Microsoft Kinect v2, HGR and NUS-II, Personal data, EgoHands, EYTH, GTEA, HOF \&, EgoHands+). The face Semantic segmentation (FASSEG) repository contains more than 500 original face images. There are six classes that are based on segmentation masks, namely mouth, nose, eyes, hair, skin, and background [68]. DATA: (MIT-CBCL, FEI, POINTING'04, SIBLINGS HQ-f).

\subsection{Identification}

Many research studies have already been done on adult image identification. Authors [69] used adult image identification depends on skin color detection. DATA: from internet.

\subsection{Blocking}

This work aims at the detection of adult images that appears on the Internet. Skin detection is of the paramount importance in detecting adult images; [70] used blocking adult images based on statistical skin detection. DATA: Compaq \& Poesia.

\subsection{Localization}

One amongst the vital pre-processing operation in every face recognition system is face localization. [28, 71] here proposed an algorithm which performs skin detection using particle swarm optimization for face localization. DATA: (FEI, LFW, Caltech \& FERET). In [72], this paper outlines another strategy for detecting the manual handmarks in 2D color images. The Handheld Destination Area is an important source of information for the detection of hand gestures. In order to properly evaluate the algorithms for hand landmark localization HGR set of hand images was used (available at http://sun.aei.polsl.pl/ mkawulok/gestures). 


\subsection{Steganography \& Cryptography}

Steganography is the art of hiding information in another medium to make mysterious correspondence. The currently used steganography technology depends on biometrics. In addition, the biometric enhancement used to update steganography is the positioning of skin tones in images [73].

In the proposed procedure [74], the biometric coverage used to make steganography is to make a skin tone region of the images. The mysterious and secret data information is embedded in ROI and not in the whole video. In [75], this article recommends a blind videotape steganography algorithm that focuses on high sensitivity and compression of MPEG-4. The proposed algorithm adds large secret pictures with video recordings. Human skin areas are selected as an ROI for the data embedding process.

Authors [76] presented an interesting strategy in which secret information can be embedded in the skin because it is not a very basic for HVS (human visual system). From the beginning, an image is taken then skin tone detection is performed based on HSV MODEL. LSB based on encoding method has been proposed in [77] that utilized DCT and pixel flipping technique to form the stego-image. Stego-key generation method is also implemented to validate the authorized receiver during retrieval. The image of the skin disease is considered to be both, cover image and stego-image. The receiver can retrieve the original image using key-based decryption algorithm, LSB, and pixel flipping method. DATA: (From internet, videos and image).

\section{RESULTS AND DISCUSSION}

There is varied amount of journals research publishers, such as Springer, IEEE, arXiv and Elsevier, that studied human skin application. We reviewed major applications of human skin from 2015-2019. Figure 2 illustrates the number of the published article based on the publisher of each human skin detection application. It can see that the Detection application has the highest number of published articles. In addition, the IEEE and Springer publisher demonstrates the highest number 23 and 20 of published articles, respectively. A large number of researches that have been published in the publishers with high reputation have found this figure. As you can see, each application has the highest amount of papers in the journal. For detection, recognition, tracking, classification and segmentation applications, IEEE is the highest number of journals with $23,11,4,4$, and 4 respectively. On the other hand, steganography application has the high amount of papers in Springer journal. Human skin detection is one of the most captivating topics in the eyes of researchers, where through Figure 2 has been found a large number of studies that have been published in the journals with high reputation.

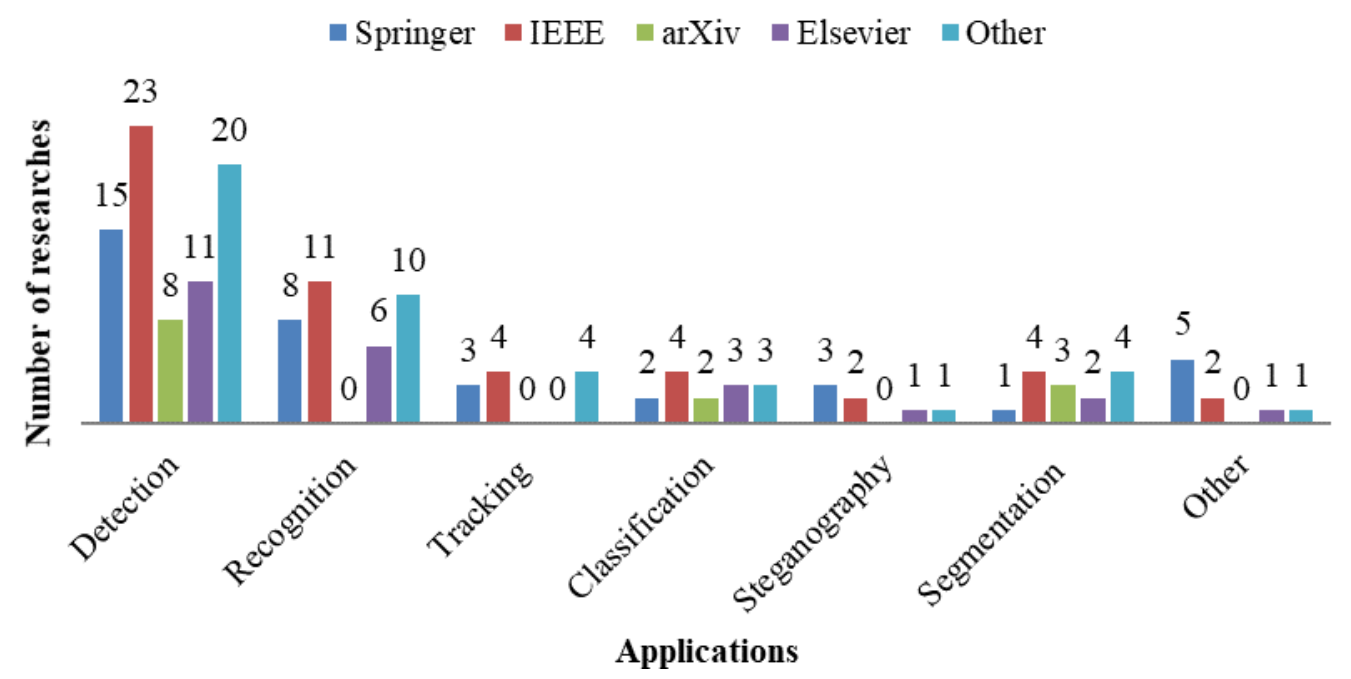

Figure 2. The number of researches in journal for human skin applications

Figure 3 has summarized the number of studies in human skin applications in 2015-2019. With a total of 149 papers, we can conclude that the applications are in the form of pie chart statistics. As we can see, the majority of researches are in detection and recognition with $43 \%$ and $28.5 \%$, respectively. However, a few studies were published for the applications of identification, blocking, and cryptography. It is noted that the detection is one of the hottest topics last five years for human skin detection. 


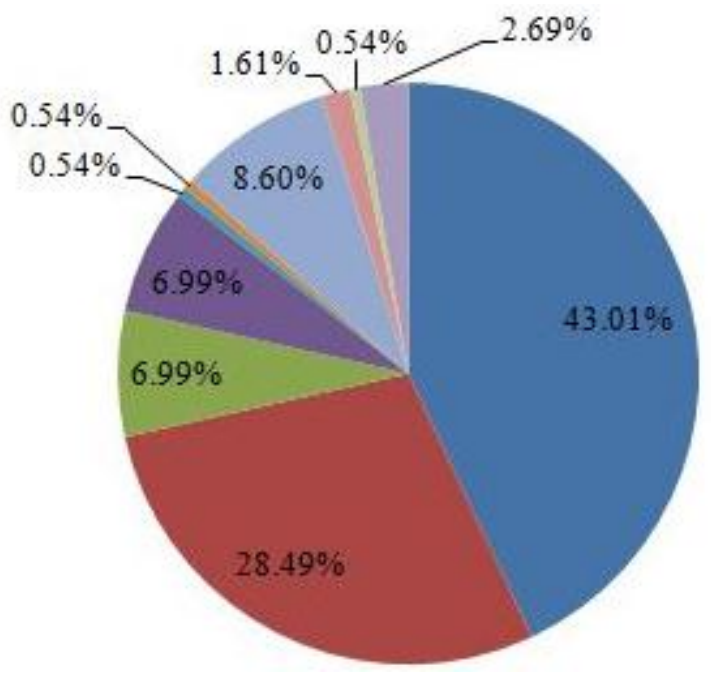

Detection

Recognition

Tracking

Segmentation

Blocking

Identification

Classification

Localization

in Cryptography

Steganography

Figure 3. The number of researches in journal for human skin applications

\section{CONCLUSION}

This article has investigated many important applications for humans and human's life (such as detection, classification, blocking, cryptography, identification, localization, steganography, segmentation, tracking, and recognition) based image processing. The investigation is implemented by searching for the human skin application within several international academic search engines, such as Springer, IEEE, arXiv and Elsevier from duration criteria of 2015-2019. Among these different applications, the skin detection is the most repetitive application published last five years in international journals. The result shows that $43 \%$ of the published articles are developed algorithms for skin detection applications. Human skin detection is very important and has many applications for society, which are important and effective for human needs and fulfil many tasks. On the other hand, each application contains many scientific fields, as every field uses a database of images that varies according to the type and need. It is worth mentioning that the studies on the human skin detection and its applications are very important where the studies have been published in international journals and publishers; such as Springer, IEEE, arXiv, and Elsevier. The investigation of this article guides the researchers for the hottest application and research scope. In short, more development is recommended on the image processing for human skin detection in recent years

\section{REFERENCES}

[1] M. R. Islam, U. K. Mitu, R. A. Bhuiyan, and J. Shin, "Hand gesture feature extraction using deep convolutional neural network for recognizing American sign language," 2018 4th International Conference on Frontiers of Signal Processing (ICFSP), pp. 115-119, 2018.

[2] S. Ahlawat, V. Batra, S. Banerjee, J. Saha, and A. K. Garg, "Hand gesture recognition using convolutional neural network," in International Conference on Innovative Computing and Communications, pp. 179-186, 2019.

[3] S. Hussain, R. Saxena, X. Han, J. A. Khan, and H. Shin, "Hand gesture recognition using deep learning," 2017 International SoC Design Conference (ISOCC), pp. 48-49, 2017.

[4] T. Sabharwal, R. Gupta, L. H. Son, R. Kumar, and S. Jha, "Recognition of surgically altered face images: an empirical analysis on recent advances," Artificial Intelligence Review, vol. 52, no. 2, pp. 1009-1040, 2019.

[5] N. Abudarham, L. Shkiller, and G. Yovel, "Critical features for face recognition," Cognition, vol. 182, pp. 73-83, 2019.

[6] N. Pratap, S. Arya, and N. Rathi, "Significance of spectral curve in face recognition," International Journal of Multidisciplinary Research, vol. 1, no. 1, pp. 51-55, 2019.

[7] Y. Wu, S. Yu, and M. Yang, "High-performance gesture recognition system," Sensors and Materials, vol. 31, no. 3, pp. 923-937, 2019.

[8] N. C. F. Codella, Q. B. Nguyen, S. Pankanti, D. Gutman, B. Helba, A. Halpern, and J. R. Smith, "Deep learning ensembles for melanoma recognition in dermoscopy images," IBM Journal of Research and Development, vol. 61, no. $4 / 5$, pp. 1-5, 2017.

[9] M. Rhanoui, S. Yousfi, M. Mikram, and H. Merizak, "Forecasting financial budget time series: ARIMA random walk vs LSTM neural network," International Journal of Artificial Intelligence, vol. 8, no. 4, pp. 317-327, 2019.

[10] P. M. Kumar, U. Gandhi, R. Varatharajan, G. Manogaran, Jidhesh R., and T. Vadivel, "Intelligent face recognition and navigation system using neural learning for smart security in internet of things," Cluster Computing, vol. 22, no. 4, pp. 7733-7744, 2019. 
[11] E-J. Cheng, K-P. Chou, S. Rajora, B-H. Jin, M. Tanveer, C-T. Lin, K-Y. Young, W-C. Lin, and M. Prasad, "Deep sparse representation classifier for facial recognition and detection system," Pattern Recognition Letters, vol. 125, pp. 71-77, 2019.

[12] F. Tang, X. Wu, Z. Zhu, Z. Wan, Y. Chang, Z. Du, and L. Gu, "An end-to-end face recognition method with alignment learning," Optik, vol. 205, p. 164238, 2020.

[13] L. B. Neto, F. Grijalva, V. R. M. L. Maike, L. C. Martini, D. Florencio, M. C. C. Baranauskas, A. Rocha, and S. Goldenstein "A kinect-based wearable face recognition system to aid visually impaired users," IEEE Transactions on Human-Machine Systems, vol. 47, no. 1, pp. 52-64, Feb. 2017.

[14] A. Ahmad, M. L. Othman, K. K. B. Zainab, H. Hizam, and N. Azis, "Adaptive ANN based differential protective relay for reliable power transformer protection operation during energisation," International Journal of Artificial Intelligence, vol. 8, no. 4, pp. 307-316, 2019.

[15] S. B. Jadhav, V. R. Udupi, and S. B. Patil, "Convolutional neural networks for leaf image-based plant disease classification," International Journal of Artificial Intelligence, vol. 8, no. 4, pp. 328-341, 2019.

[16] H. A. Haenssle, C. Fink, R. Schneiderbauer, F. Toberer, T. Buh, A. Blum, A. Kalloo, A. Ben Hadj Hassen, L. Thomas, A. Enk, and L. Uhlmann, "Man against machine: diagnostic performance of a deep learning convolutional neural network for dermoscopic melanoma recognition in comparison to 58 dermatologists," Annals of Oncology, vol. 29, no. 8, pp. 1836-1842, 2018.

[17] V. Kumar and T. Choudhury, "Real-time recognition of malignant skin lesions using ensemble modeling," Journal of Scientific and Industrial Research, vol.78, no. 3, pp. 148-153, 2019.

[18] V. K. Verma, S. Srivastava, T. Jain, and A. Jain, "Local invariant feature-based gender recognition from facial images," Soft Computing for Problem Solving, vol. 2, pp. 869-878, 2019.

[19] M. B. Garcia, T. F. Revano, B. G. M. Habal, J. O. Contreras, and J. B. R. Enriquez, "A pornographic image and video filtering application using optimized nudity recognition and detection algorithm," 2018 IEEE 10th International Conference on Humanoid, Nanotechnology, Information Technology, Communication and Control, Environment and Management (HNICEM), pp. 1-5, 2018.

[20] L. Zhuo, Z. Geng, J. Zhang, and X. guang Li, "ORB feature based web pornographic image recognition," Neurocomputing, vol. 173, no. 3, pp. 511-517, 2016.

[21] R. Zhu, X. Wu, B. Zhu, and L. Song, "Application of pornographic images recognition based on depth learning," Proceedings of the 2018 International Conference on Information Science and System, pp. 152-155, 2018.

[22] M. A. A. Milton, "Automated skin lesion classification using ensemble of deep neural networks in ISIC 2018: Skin lesion analysis towards melanoma detection challenge," arXiv preprint arXiv:1901.10802, 2019.

[23] N. Codella, V. Rotemberg, P. Tschandl, M. E. Celebi, S. Dusza, D. Gutman, B. Helba, A. Kalloo, K. Liopyris, M. Marchetti, H. Kittler, and A. Halpern, "Skin lesion analysis toward melanoma detection 2018: A challenge hosted by the international skin imaging collaboration (ISIC)," arXiv preprint arXiv:1902.03368, 2019.

[24] G. Arora, A. K. Dubey, and Z. A. Jaffery, "Classifiers for the detection of skin cancer," Smart Computing and Informatics, pp. 351-360, 2018.

[25] P. M. Burlina, N. J. Joshi, E. Ng, S. D. Billings, A. W. Rebman, and J. N. Aucott, "Automated detection of erythema migrans and other confounding skin lesions via deep learning," Computers in Biology and Medicine, vol. 105, pp. 151-156, 2019.

[26] M. A. A. Akash, M. A. H. Akhand, and N. Siddique, "Robust face detection using hybrid skin color matching under different illuminations," 2019 Int. Conf. on Electrical, Comp. and Comm. Eng. (ECCE), pp. 1-6, 2019.

[27] M. Zollhöfer, J. Thies, P. Garrido, D. Bradley, T. Beeler, P. Pérez, M. Stamminger, M. Nießner, and C. Theobalt, "State of the art on monocular 3D face reconstruction, tracking, and applications," Computer Graphics Forum, vol. 37, no. 2, pp. 523-550, 2018.

[28] R. Ramesh, A. C. Kulkarni, N. R. Prasad, and K. Manikantan, "Face recognition using snakes algorithm and skin detection based face localization," in Proceedings of the International Conference on Signal, Networks, Computing, and Systems, pp. 61-71, 2017.

[29] V. Singh and D. Aswani, "Face detection in hybrid color space using HBF-KNN," in Proceedings of International Conference on Recent Advancement on Computer and Communication, pp. 489-498, 2018.

[30] X. Deng, Y. Zhang, S. Yang, P. Tan, L. Chang, Y. Yuan, and H. Wang, "Joint hand detection and rotation estimation using CNN," IEEE Transactions on Image Processing, vol. 27, no. 4, pp. 1888-1900, April 2018.

[31] S. Bambach, S. Lee, D. J. Crandall, and C. Yu, "Lending a hand: Detecting hands and recognizing activities in complex egocentric interactions," 2015 IEEE Int. Conference on Computer Vision (ICCV), pp. 1949-1957, 2015.

[32] T. H. N. Le, K. G. Quach, C. Zhu, C. N. Duong, K. Luu, and M. Savvides, "Robust hand detection and classification in vehicles and in the wild," 2017 IEEE Conference on Computer Vision and Pattern Recognition Workshops (CVPRW), pp. 1203-1210, 2017.

[33] K K. Roy, A. Mohanty, and R. R. Sahay, "Deep learning based hand detection in cluttered environment using skin segmentation," 2017 IEEE International Conference on Computer Vision Workshops (ICCVW), pp. 640-649, 2017.

[34] Q. Wang, G. Zhang, and S. Yu, "2D hand detection using multi-feature skin model supervised cascaded CNN," Journal of Signal Processing Systems, vol, 91, no. 10, pp. 1105-1113, 2019.

[35] D. Ganguly, M. H. Mofrad, and A. Kovashka, "Detecting sexually provocative images," 2017 IEEE Winter Conference on Applications of Computer Vision (WACV), pp. 660-668, 2017.

[36] C. Tian, X. Zhang, W. Wei, and X. Gao, "Color pornographic image detection based on color-saliency preserved mixture deformable part model," Multimedia Tools and Applications, vol. 77, no. 6, pp. 6629-6645, 2018.

[37] F. Nian, T. Li, Y. Wang, M. Xu, and J. Wu, "Pornographic image detection utilizing deep convolutional neural networks," Neurocomputing, vol. 210, pp. 283-293, 2016. 
[38] J. Yan and X. Gao, "Pornographic video detection with MapReduce," International Journal of Machine Learning and Cybernetics, vol. 9, no. 12, pp. 2105-2115, 2018.

[39] C. Caetano, S. Avila, W. R. Schwartz, S. J. F. Guimarães, and A. de A. Araújo, "A mid-level video representation based on binary descriptors: A case study for pornography detection," Neurocomputing, vol. 213, pp. 102-114, 2016.

[40] M. Perez, S. Avila, D. Moreira, D. Moraes, V. Testoni, E. Valle, S. Goldenstein, and A. Rocha, "Video pornography detection through deep learning techniques and motion information," Neurocomputing, vol. 230, pp. 279-293, 2017.

[41] A. Gangwar, E. Fidalgo, E. Alegre, and V. González-Castro, "Pornography and child sexual abuse detection in image and video: A comparative evaluation," 8th International Conference on Imaging for Crime Detection and Prevention (ICDP 2017), pp. 37-42, 2017.

[42] Y. Liu, L. Nie, L. Liu, and D. S. Rosenblum, "From action to activity: sensor-based activity recognition," Neurocomputing, vol. 181, pp. 108-115, 2016.

[43] L. Maleš, D. Marčetić, and S. Ribarić, "A multi-agent dynamic system for robust multi-face tracking," Expert Systems with Applications, vol. 126, pp. 246-264, 2019.

[44] H. Singh and E. S. Sehgal, "Face detection and tracking using combination of sdm viola jones and skin color based segmentation," International Journal of Engineering Development and Research, vol. 5, no. 2, pp. 1717-1722, 2017.

[45] M. S. Al-Ani, "Happiness measurement via classroom based on face tracking," UHD Journal of Science and Technology, vol. 3, no. 1, pp. 9-18, 2019.

[46] T. Li, P. Zhou, and H. Liu, "Multiple features fusion based video face tracking," Multimedia Tools and Applications, vol. 78, no. 15, pp. 21963-21980, 2019.

[47] F. Dadgostar and A. Sarrafzadeh, "Gesture-based human-machine interfaces: a novel approach for robust hand and face tracking," Iran Journal of Computer Science, vol. 1, no. 1, pp. 47-64, 2018.

[48] L. Cuimei, Q. Zhiliang, J. Nan, and W. Jianhua, "Human face detection algorithm via Haar cascade classifier combined with three additional classifiers," 2017 13th IEEE International Conference on Electronic Measurement \& Instruments (ICEMI), pp. 483-487, 2017.

[49] H.-S. Yeo, B.-G. Lee, and H. Lim, "Hand tracking and gesture recognition system for human-computer interaction using low-cost hardware," Multimedia Tools and Applications, vol. 74, no. 8, pp. 2687-2715, 2015.

[50] D. Mazumdar, A. K. Talukdar, and K. K. Sarma, "Gloved and free hand tracking based hand gesture recognition," 2013 1st International Conference on Emerging Trends and Applications in Computer Science, pp. 197-202, 2013.

[51] S. S. Han, M. S. Kim, W. Lim, G. H. Park, I. Park, and S. E. Chang, "Classification of the clinical images for benign and malignant cutaneous tumors using a deep learning algorithm," Journal of Investigative Dermatology, vol. 138 , no. 7 , pp. 1529-1538, 2018.

[52] Y. Liang, L. Sun, W. Ser, F. Lin, S. T. G. Thng, Q. Chen, and Z. Lin, "Classification of non-tumorous skin pigmentation disorders using voting based probabilistic linear discriminant analysis," Computers in Biology and Medicine, vol. 99, pp. 123-132, 2018.

[53] D. Bisla, A. Choromanska, J. A. Stein, D. Polsky, and R. Berman, "Skin lesion segmentation and classification with deep learning system," CoRR, 2019.

[54] M. Nasir, M. Attique Khan, M. Sharif, I. U. Lali, T. Saba, and T. Iqbal, "An improved strategy for skin lesion detection and classification using uniform segmentation and feature selection based approach," Microscopy Research and Technique, vol. 81, no. 6, pp. 528-543, 2018.

[55] A. R. Patil and S. Subbaraman, "Illumination invariant hand gesture classification against complex background using combinational features," International Journal of Computer Science and Information Security, vol. 16, no. 3, pp. 63-70, 2018.

[56] J. S. Alarifi, M. Goyal, A. K. Davison, D. Dancey, R. Khan, and M. H. Yap, "Facial skin classification using convolutional neural networks," in International Conference Image Analysis and Recognition, pp. 479-485, 2017.

[57] K. Zhou, L. Zhuo, Z. Geng, J. Zhang, and X. G. Li, "Convolutional neural networks based pornographic image classification,” 2016 IEEE Second International Conference on Multimedia Big Data (BigMM), pp. 206-209, 2016.

[58] J. Jagtap and M. Kokare, "Human age classification using appearance and facial skin ageing features with multiclass support vector machine," International Journal of Biometrics, vol. 11, no. 1, pp. 22-34, 2019.

[59] T. Akram, M. A. Khan, M. Sharif, and M. Yasmin, "Skin lesion segmentation and recognition using multichannel saliency estimation and M-SVM on selected serially fused features," Journal of Ambient Intelligence and Humanized Computing, pp. 1-20, 2018.

[60] R. Sumithra, M. Suhil, and D. S. Guru, "Segmentation and classification of skin lesions for disease diagnosis," Procedia Computer Science, vol. 45, pp. 76-85, 2015.

[61] C. Kaul, S. Manandhar, and N. Pears, "Focusnet: An attention-based fully convolutional network for medical image segmentation," 2019 IEEE 16th International Symposium on Biomedical Imaging (ISBI 2019), pp. 455-458, 2019.

[62] R. C. Hardie, R. Ali, M. S. De Silva, and T. M. Kebede, "Skin lesion segmentation and classification for ISIC 2018 using traditional classifiers with hand-crafted features," arXiv preprint arXiv:1807.07001, 2018.

[63] G. Kaur and K. Joshi, "Automatic detection and segmentation of skin melanoma images-an introduction," International Journal of Emerging Research in Management \& Technology, vol. 4, no. 9, pp. 120-123 2015.

[64] B. Kang, K-H. Tan, N. Jiang, H-S. Tai, D. Tretter, and T. Nguyen, "Hand segmentation for hand-object interaction from depth map," 2017 IEEE Global Conf. on Signal and Information Processing (GlobalSIP), pp. 259-263, 2017.

[65] Q. Zhang, M. Yang, K. Kpalma, Q. Zheng, and X. Zhang, "Segmentation of hand posture against complex backgrounds based on saliency and skin colour detection," IAENG International Journal of Computer Science, vol. 45 , no. 3, pp. 435-444, 2018.

[66] A. U. Khan and A. Borji, "Analysis of hand segmentation in the wild," 2018 IEEE/CVF Conference on Computer Vision and Pattern Recognition, pp. 4710-4719, 2018. 
[67] S. Gong, G. Li, Y. Zhang, C. Li, and L. Yu, "Application of static gesture segmentation based on an improved canny operator," The Journal of Engineering, vol. 2019, no. 15, pp. 543-546, 2019.

[68] S. Benini, K. Khan, R. Leonardi, M. Mauro, and P. Migliorati, "FASSEG: a FAce Semantic SEGmentation repository for face image analysis," Data in Brief, vol. 24, p. 103881, 2019.

[69] R. Balamurali and A. Chandrasekar, "Multiple parameter algorithm approach for adult image identification," Cluster Computing, vol. 22, no. 5, pp. 11909-11917, 2019.

[70] H. Zheng and M. Daoudi, "Blocking adult images based on statistical skin detection," Electronic Letters on Computer Vision and Image Analysis, vol. 4, no. 2, pp. 1-14, 2004.

[71] S. Jois, R. Ramesh, and A. C. Kulkarni, "Face localization using skin colour and maximal entropy based particle swarm optimization for facial recognition," 2017 4th IEEE Uttar Pradesh Section International Conference on Electrical, Computer and Electronics (UPCON), pp. 156-161, 2017.

[72] T. Grzejszczak, M. Kawulok, and A. Galuszka, "Hand landmarks detection and localization in color images," Multimedia Tools and Applications, vol. 75, no. 23, pp. 16363-16387, 2016.

[73] A. A. Shejul and U. L. Kulkarni, "A secure skin tone based steganography using wavelet transform," International Journal of Computer Theory and Engineering, vol. 3, no. 1, pp. 16-22, 2011.

[74] P. Kumar and K. Singh, "An improved data-hiding approach using skin-tone detection for video steganography," Multimedia Tools and Applications, vol. 77, no. 18, pp. 24247-24268, 2018.

[75] M. M. Sadek, A. S. Khalifa, and M. G. M. Mostafa, "Robust video steganography algorithm using adaptive skin-tone detection," Multimedia Tools and Applications, vol. 76, no. 2, pp. 3065-3085, 2017.

[76] M. Kude and M. Borse, "Skintone detection-based steganography using wavelet transform," 2016 International Conference on Automatic Control and Dynamic Optimization Techniques (ICACDOT), pp. 440-443, 2016.

[77] K. Hossain, S. Jana, S. Mukherjee, and R. Parekh, "Secured transmission of sensitive images of skin diseases using steganography and cryptography," 2017 IEEE Calcutta Conference (CALCON), pp. 95-99, 2017. 